\title{
RELATIONSHIP BETWEEN TILS AND NEOADJUVANT CHEMOTHERAPY RESPONSE IN BREAST CANCER
}

Fabio Postiglione Mansani', Hanna Jeny Schimim²

${ }^{1}$ Universidade Estadual de Ponta Grossa - Ponta Grossa (PR), Brasil.

${ }^{2}$ Hospital Universitário Regional dos Campos Gerais - Ponta Grossa (PR), Brazil.

Introduction: Breast cancer corresponds to $25 \%$ of new cancer cases each year. In Brazil, the rate is $29.5 \%$ and 59,700 new cases were estimated in 2019. Tumor infiltrating lymphocytes (TIL) are observed in some solid tumors, including breast cancer, the amount of TIL is an indirect marker of the pre-existing antitumor activated T cell response, reflecting the host's antitumor immunity. Studies show that its presence is an independent predictor of complete pathological response (PRC) to neoadjuvant chemotherapy (QTneo) and its presence provides a favorable prognosis in breast cancer. Objectives: To evaluate the relationship between the presence and value of TIL with the pathological response after QTneo in patients with breast cancer. Materials and methods: An analytical, descriptive, retrospective, case-control study was carried out with 40 patients diagnosed with breast cancer who underwent QTneo between 01/01/2016 to 01/01/2019 at the ISPON complex in the city of Ponta Grossa, Paraná. Data collection was carried out in electronic medical records. The association between variables was tested with the $\rrbracket^{2}$ test and the intensity of the association with the Odds Ratio estimate with a $95 \%$ confidence interval. TIL were considered absent when 0 , low when $1 \%$ to $10 \%$, moderate when $11 \%$ to $59 \%$, and high if $60 \%$ or more; this division was based on other articles that quantify TIL. Results: The mean age of the patients in the study was 50 years old, 13 of them (32.5\%) had PRC, 15 (37.5\%) had a partial response, and 13 (30\%) had no response to QTneo. Of the 13 that obtained PRC, 7 (53.8\%) were triple negative (TN), 5 (38.4\%) HER 2, and 1 (7.6\%) Luminal. PRC was more frequent in triple negative invasive ductal carcinoma (53.8\%), the frequency of PRC in the luminal subtype was significantly lower than that of TN ( $\mathrm{p}=0.03$ ), with the worst response to QTneo. The mean age of patients who had PRC was 48 years. TIL were present in 27 (67.5\%) patients, whose value was low in 57.5\%, moderate in 3 patients, and high in one case. The presence of TIL, regardless of their value, increased the probability of PRC by 7.7 times when compared to their absence (OR 7.7 CI 1.15-51.17 p=0.03). When considering its value, it was found that higher values conferred a significant association with PRC ( $\mathrm{p}=0.01)$. There was a statistically significant association between the clinical stage (CS) and the presence of response to QTneo, patients with locally advanced neoplasia were 6.44 times more likely to respond than patients in an early stage $(\mathrm{p}=0.015)$. Conclusion: TIL can be used as a predictor of response to QTneo, being an aid in choosing this treatment and an indicator of better prognosis. The evaluation of TIL should be performed routinely in the preparation of anatomopathological reports, as it is an indication of the investigation of PD-L1 and its blockade, if present. 